九州大学学術情報リポジトリ

Kyushu University Institutional Repository

\title{
RELIABILITY IN CONCURRENT SCIENTIFIC COMPUTING FOR VOLTERRA TYPE EQUATIONS
}

Dobner, Hans-Jurgen

Mathemat ischess Institut II, Universitat Karlsruhe

https://doi.org/10.5109/13476

出版情報: Bulletin of informatics and cybernetics. 31 (1), pp.1-14, 1999-03. Research Association of Statistical Sciences

バージョン :

権利関係 : 


\title{
RELIABILITY IN CONCURRENT SCIENTIFIC COMPUTING FOR VOLTERRA TYPE EQUATIONS
}

By

\author{
Hans-J ürgen DoBNER*
}

\begin{abstract}
We deal with an efficient approach for the complete error control in scientific computing. For results obtained by standard numerical routines we provide in an additional validation step existence and enclosure of the corresponding solutions. The basic ideas of these methods are derived exemplary for nonlinear Volterra equations and then generalized to various initial value problems. We combine principles from functional analysis and enclosure theory to determine realistic and reliable a posteriori error bounds for numerical approximations. This method has been applied successfully to various problems.
\end{abstract}

Key words : Scientific computing, reliable error bounds, Volterra equations, initial value problems.

AMS Subject Classification : 34-04, 35-04, 45D05, 65G99, 65L05, 65M15, 65R10.

\section{The Problem}

Scientific computing focuses on the algorithmic structure of numerical methods and merges numerical analysis to develop solution methods and computer science to develop effective implementations. Nevertheless a shortcoming of contemporary scientific computing is the lack of computable realistic and safe error bounds for numerical solutions. This paper derives a concept to reach this goal in the framework of scientific computing. Since the early 80 's, a precise computer arithmetic is realized on several platforms (cf. Kulisch and Miranker (1981)), providing a basis for a rigorous software assisted error treatment. There are two different principles in order to estimate all kind of computational errors. The first one is to construct new algorithms. Most of the papers dealing with reliability in scientific computing use this way to attack the problem. For finite dimensional systems see for example Rump (1993), Neumaier (1990). For infinite dimensional systems see e.g. Lohner (1988), Kaucher and Miranker (1984), Dobner $(1996,1993)$. The second way is to use a traditional scheme and to compute in an additional step safe error bounds for a numerical solution obtained by such a scheme.

\footnotetext{
* Mathematisches Institut II, Universität Karlsruhe, Englerstr. 2, D-76128 Karlsruhe. e-mail: dobner@ ma2mar1.mathematik.uni-karlsruhe.de
} 
The latter procedure is more effective because a large number of numerical algorithms can be employed and only the validation step must be carried out independent of the specific package used. But the determination of computable, close and mathematically guaranteed bounds on an approximation poses some difficult questions and is therefore often ignored in scientific computing. Therefore only few papers use this approach (cf. Linz (1985), Plum (1994), Nakao (1988, 1992, 1994)). For deriving our methodology which is based upon the approach proposed by Plum (1994), we turn our attention to the nonlinear Volterra equation of the second kind

$$
y(s)=g(s)+\int_{0}^{s} k(s, t, y(t)) d t=: f(y), \quad 0 \leq s \leq a .
$$

We assume that kernel and forcing term in (1.1) are continuous real functions. Additionally the kernel should be continuously differentiable with respect to the third variable. In short notation (1.1) is written as (we use for kernel and corresponding operator the same symbol)

$$
y=g+k(y) \quad \text { or } \quad(I-k) y=g .
$$

As underlying space we take $\mathcal{B}=C[0, a]$, which is, equipped with the maximum norm

$$
\|u\|_{\infty}:=\max _{0 \leq s \leq a}|u(s)|
$$

a Banach space.

Before going into detail we summarize the underlying principles of computer assisted error computation.

\section{Foundations of Computer Assisted Error Control}

For a safe error control we have to take into account both - roundoff and approximation-errors. Intervals are an efficient tool to describe such imprecisions. We consider real intervals of the form

$$
A=[a]=[\underline{a}, \bar{a}]=\{s \in \mathbb{R} \mid \underline{a} \leq s \leq \bar{a}\} ;
$$

the set of all such intervals is denoted with $I(I R)$. For intervals the basic arithmetic operations $*=+,-, \cdot, /$ are defined according to

$$
A * B=\left[\min _{a \in A, b \in B}\{a * b\}, \max _{a \in A, b \in B}\{a * b\}\right],
$$

(cf. also Alefeld and Herzberger (1983) or Moore (1966)). In a computational framework the endpoints in (2.1) are floating point numbers. Set theoretic relations $=, \subseteq, \cup$ are explained as usual. For interval vectors and matrices these definitions apply componentwise. Some important definitions are summarized below

$$
\operatorname{mid}(A):=\frac{1}{2}(\underline{a}+\bar{a})
$$




$$
\begin{gathered}
\operatorname{diam}(A):=\bar{a}-\underline{a}, \\
|A|:=\max \{|\underline{a}|,|\bar{a}|\} .
\end{gathered}
$$

Unfortunately the distributive law is not valid in $I(I R)$, only subdistributivity holds

$$
A(B+C) \subseteq A B+A C, \quad A, B, C \in I(\mathbb{R}) .
$$

Additionally $\mathcal{B}$ is a real ordered Banach space, where the relation $\leq$ is compatible with the underlying norm $\|.\|_{\infty}$. For elements of $\mathcal{B}$ some operations $\mathcal{O}$ are available

$$
\mathcal{O}=\left\{+,-, \cdot, /, \int\right\}
$$

Let $\mathcal{S}_{n}=\operatorname{span}\left(b_{1}, \ldots, b_{n}\right)$ be a finite dimensional subspace of $\mathcal{B}$, then we consider special sets, such called function intervals, having the form

$$
J_{n}:=\left\{H:=[\underline{h}, \bar{h}] \mid \underline{h}, \bar{h} \in \mathcal{S}_{n}\right\} .
$$

REMARK 2.1. Due to construction all elements of $J_{n}$ are closed, bounded and convex.

REMARK 2.2. For elements of $J_{n}$ the definitions of midpoint, diameter are explained analogously.

Additionally we define for $F \in J_{n}$

$$
\|F\|=\sup _{f \in F}\|f\|_{\infty}
$$

The operations $* \in \mathcal{O}$ are modified to $\hat{*}$ for elements of $J_{n}$, such that $J_{n}$ is algebraically closed under $\hat{\mathcal{O}}$ :

$$
\left\{\begin{array}{l}
U \hat{*} V=: W \\
\text { where } \\
W:=\min _{\operatorname{diam}(X),}\left\{X \in J_{n} \mid U * V \subseteq X\right\}, \quad U, V \in J_{n} .
\end{array}\right.
$$

For $f \in \mathcal{B}$, a function interval $F \in J_{n}$ is called enclosure of $f$ (in sign $F \in \mathcal{E}(f)$ ) iff

$$
f(s) \in F(s), \quad 0 \leq s \leq a
$$

\section{An Estimation Theorem}

Before going into detail we figure out first the basic ideas of our method. This is accomplished by explaining the manipulations referencing to the corresponding formula below. Thus the reader is able to evaluate the proposed strategy. Considered problems are initial value problems. First a numerical approximation $\tilde{y}$ is computed with a suitable algorithm. At this point, usual calculations terminate and the accuracy question remains 
open. In addition to common practice, we continue by computing guaranteed estimates on $\tilde{y}$. To this avail in (3.1)-(3.3) the original question is formulated as root finding problem. Applying the Newton operator, it is then rewritten as fixed point equation with a compact operator, this is performed in (3.4)-(3.8). The basic idea, worked out in (3.9)-(3.14), is to construct, starting from $\tilde{y}$, a set $W$, which is mapped by this compact operator into itself. Schauder's fixed point principle assures that the true solution lies within $W$ (see (3.15)) and yields at the same time error bounds. Rewrite (1.1) as root finding problem

$$
l(y):=y-f(y)=0,
$$

and apply the Newton operator

$$
y=y-\left(l^{\prime}(\eta)\right)^{-1} l(y),
$$

where the Fréchet derivative $l^{\prime}(\eta)$ at $\eta$ is:

$$
\begin{aligned}
R(u) & :=l^{\prime}(\eta)(u)=u(s)-\int_{0}^{s} \frac{\partial k(s, t, u)}{\partial u}(\eta) u(t) d t \\
& =: u(s)-\int_{0}^{s} p(s, t ; \eta) u(t) d t, \quad 0 \leq s \leq a .
\end{aligned}
$$

We use the same notation for both kernel and operator

$$
p_{\eta}(u)=\int_{0}^{1} p(s, t ; \eta) u(t) d t, \quad 0 \leq s \leq a
$$

Thus (3.1) becomes

$$
\begin{aligned}
y & =R^{-1}(R(y)-(y-f(y))) \\
& =R^{-1}\left(y-p_{\eta}(y)-(y-f(y))\right) \\
& =R^{-1}\left(f(y)-p_{\eta}(y)\right)=: T(y)
\end{aligned}
$$

With an approximation $\tilde{y}$, introduce the operator

$$
T_{m}(y):=T(\tilde{y}+y)-\tilde{y}
$$

thus a fixed point $\hat{y}$ of $T_{m}$ provides $\tilde{y}+\hat{y}$ as a fixed point of $T$ and vice versa. Consequently instead of solutions for (1.1), which are close to $\tilde{y}$, one looks for fixed points of $T_{m}$ which are close to 0 . Constructed set is a neighbourhood $V$ of 0 with

$$
T_{m}(V) \subseteq V
$$

If such a set has been achieved then $W:=V+\tilde{y}$ fulfills

$$
T(W) \subseteq W
$$


In the further considerations we take always $\tilde{y}$ for $\eta$, therefore the index $p_{\eta}=p_{\tilde{y}}$ can be dropped for sake of clearity. We rewrite $T_{m}(y)=y$ in the form

$$
\begin{aligned}
y & =R^{-1}[f(\tilde{y}+y)-p(\tilde{y}+y)]-\tilde{y} \\
& =R^{-1}[f(\tilde{y}+y)-p(\tilde{y})-p(y)-(\tilde{y}-p(\tilde{y}))] \\
& =R^{-1}[f(\tilde{y})-\tilde{y}+f(\tilde{y}+y)-f(\tilde{y})-p(y)]
\end{aligned}
$$

Using the shortening

$$
\begin{gathered}
d(\tilde{y}):=f(\tilde{y})-\tilde{y} \\
q(y):=f(\tilde{y}+y)-f(\tilde{y})-p(y)
\end{gathered}
$$

equation (3.7) becomes

$$
y=R^{-1}(d(\tilde{y})+q(y)) .
$$

The idea is to construct a nonvoid set $V$ such that

$$
R^{-1}(d(\tilde{y})+q(V)) \subseteq V .
$$

With $\gamma \in(0, \infty)$ we specify $0 \in V \in J_{n}$ through

$$
\operatorname{diam}(V) \leq \gamma
$$

THEOREM 3.1. Let (3.9) be true for $V \in J_{n}$, then the existence of a fixed point $\hat{y}$ of problem (3.7) is ensured, additionally the corresponding solution is enclosed within

$$
\hat{y} \in V \text {. }
$$

Proof. By construction $R^{-1}(d(\tilde{y})+q(\cdot))$ is a compact operator, so Schauder's fixed point principle applies assuring the existence of a fixed point within the set $V$.

We construct $V$ by bounding the terms occuring in (3.9) normwise. Due to our assumptions there exist nonnegative real constants $\rho, \delta, \psi$ such that

$$
\begin{gathered}
\left\|R^{-1}\right\| \leq \rho, \\
\|d(\tilde{y})\| \leq \delta, \\
\|q(V)\| \leq \psi(V) .
\end{gathered}
$$


THEOREM 3.2. If for $\gamma>0$ the inequality

$$
0 \leq \delta \leq \frac{\gamma}{\rho}-\psi(\gamma)
$$

holds, then the solution of (1.1) suffices

$$
\|y-\tilde{y}\| \leq \gamma
$$

Proof. We have

$$
\begin{gathered}
\left\|R^{-1}(d(\tilde{y})+q(v))\right\| \leq\left\|R^{-1}\right\|(\|d(\tilde{y})\|+\|q(v)\|) \\
\leq \rho(\delta+\psi(\gamma))
\end{gathered}
$$

therefore, enclosure (3.9) is attained when (3.14) is true. The rest is a consequence of (3.4) and (3.6).

REMARK 3.3. In the linear case, we get an a posteriori bound for (1.2) simply by

$$
\|y-\tilde{y}\| \leq\left\|(I-k)^{-1}\right\|\|d(\tilde{y})\|
$$

\section{Computation of the Necessary Bounds}

In this section we emphasize to compute the necessary constants $\rho, \delta$ and $\psi$.

\subsection{Calculation of $\rho$}

Since $\rho_{\eta}$ is a linear Volterra operator of the second kind, the inverse operator of $R=\left(I-\rho_{\eta}\right),(I$ denotes the identity $)$ is given as a Neumann series

$$
R^{-1}=\left(I-\rho_{\eta}\right)^{-1}=\sum_{\nu=0}^{\infty} \rho_{\eta}^{\nu}
$$

satisfying

$$
\left\|R^{-1}\right\| \leq \exp (M a)=: \rho
$$

where the constant $M$ is given by

$$
|p(s, t)| \leq M, \quad 0 \leq s, t \leq a .
$$

The iterated operators $\rho_{\eta}^{\nu}$ are recursively defined by $\rho_{\eta}^{0}:=I$ and $\rho_{\eta}^{\nu}:=\rho_{\eta} \rho_{\eta}^{\nu-1}$ for $\nu \in \mathbb{N}$. 


\subsection{Calculation of $\delta$}

A safe bound on the defect is materialized with extensive use of enclosure mathematics. Let $\tilde{y}$ be an initial approximation, and $\tilde{Y} \in \mathcal{E}(\tilde{y}), G \in \mathcal{E}(g), K \in \mathcal{E}(k)$ be corresponding enclosures (for technical details we refer to $\S 7$ ), then we have

$$
d(\tilde{Y}) \in \tilde{Y}-F(\tilde{Y})
$$

and consequently

$$
\|d(\tilde{Y})\| \leq\|\tilde{Y}-F(\tilde{Y})\|=: \delta
$$

\subsection{Calculation of $\psi(\gamma)$}

Due to the regularity of $k$ and $p$ we have

$$
\begin{gathered}
f(\tilde{y}+y)-f(\tilde{y})-p(y)= \\
\left(g(s)+\int_{0}^{s} k(s, t, \tilde{y}(t)+y(t)) d t-\left(g(s)+\int_{0}^{s} k(s, t, \tilde{y}(t)) d t\right)\right. \\
-\int_{0}^{s} \frac{\partial k(s, t, u)}{\partial u}(\tilde{y}(t)) y(t) d t \\
=\int_{0}^{s} \frac{\partial^{2} k(s, t, u(t))}{\partial u^{2}}(\xi(t)) \frac{(y(t))^{2}}{2}
\end{gathered}
$$

with an intermediate value $\xi$ between $\tilde{y}$ and $y$, thus $\xi(t) \in V$ and

$$
\psi(\gamma):=\left|\int_{0}^{s} \frac{\partial^{2} k(s, t, u)}{\partial u^{2}}(\gamma) \frac{1}{2} \gamma^{2} d t\right|
$$

fulfills (3.13).

\section{Some Generalizations}

The extension of the foregoing results to systems is possible with minor changes in notation. Using the usual vector-matrix notation the system

$$
y_{i}(s)=g_{i}(s)+\int_{0}^{s} \sum_{j=1}^{n} k_{i j}\left(s, t, y_{j}(t)\right) d t, \quad 0 \leq s \leq a, \quad i=1, \ldots, n,
$$

can be written as

$$
Y=G+\int_{0}^{s} K(s, t, Y(t)) d t, \quad 0 \leq s \leq a
$$


With this notation the results for systems are formally the same as those for single equations.

The reduction of integrodifferential equations to a system of integral equations is helpful for treating problems of the form

$$
\begin{gathered}
y^{\prime}(s)=g(s)+\int_{0}^{s} k(s, t, y(t)) d t, \quad 0 \leq s \leq a, \\
y(0)=y_{0} .
\end{gathered}
$$

An application to some problems of the first kind

$$
\int_{0}^{s} k(s, t, y(t)) d t=g(s), \quad 0 \leq s \leq a
$$

is possible, when we assume that the kernelfunction in (5.1) is a product kernel of the special type

$$
k(s, t, u)=k_{1}(s, t) k_{2}(u),
$$

where $k_{1}(s, t)$ is continuous for all $0 \leq t \leq s \leq a$ and $k_{2}^{\prime}(u)$ is continuous for all $-\infty<u<\infty$, so that with some positive constant $\alpha$

$$
\left\|k_{2}^{\prime}(u)\right\| \leq \alpha\|u\| .
$$

If furthermore

$$
g(0)=0 \quad \text { and } \quad k_{1}(s, s) \neq 0, \quad 0 \leq s \leq a,
$$

then problem (5.1) can be converted into an equivalent equation of the second kind:

$$
k_{2}(x(s))=\frac{g^{\prime}(s)}{k_{1}(s, s)}-\int_{0}^{s} \frac{1}{k_{1}(s, s)} \frac{\partial k_{1}(s, t)}{\partial s} k_{2}(x(t)) d t, \quad 0 \leq s \leq a .
$$

\section{Application to Initial Value Problems} problem

We use Volterra equations in the study of differential equations. The initial value

$$
\begin{aligned}
y^{\prime}(s) & =f(s, y(s)), \quad s \geq 0 \\
y(0) & =y_{0}
\end{aligned}
$$

is reformulated as integral equation

$$
y(s)=y_{0}+\int_{0}^{s} f(t, y(t)) d t, \quad 0 \leq s \leq a .
$$


Similarly to initial value problems, nonlinear wave equations are recasted as integral equations. Consider the hyperbolic problem

$$
\begin{array}{cc}
u_{s t}=r\left(s, t, u, u_{s}, u_{t}\right) & , \quad(s, t) \in D \subseteq \mathbb{R}^{2} \\
\left.u\right|_{\hat{D}}=b(s, t) \quad, \quad \hat{D} \subseteq \partial D,
\end{array}
$$

where the domain $D$ and a subset $\hat{D}$ of the boundary are chosen corresponding to the special initial value problem, e.g. $D$ is a rectangle if $u$ is prescribed on the two lines $s=0$ and $t=0$. As integrodifferential equation (6.3) is written as

$$
u(s, t)=g(s, t)+\iint_{D(s, t)} r\left(s, t, u, u_{s}, u_{t}\right) d D,
$$

where $g$ is a term resulting from the initial condition. This equation is treated further with the techniques described in part 5.

\section{Aspects of Implementation}

In this section, we give a short description about tools, needed for the implementation of the method proposed in the previous sections.

\subsection{Computational tools}

The tools, necessary for the calculation of safe bounds in scientific computing are available as software packages (see Hammer et al. (1993)).

\section{Interval extensions}

For a real valued quantity the problem arises to assign an enclosure $F \in \mathcal{E}(f)$ with small width. $F$ is gained by computing a truncated Taylor- or Fourier series and bounding the remainder term. In remainder terms a variable $\xi$ is often involved, whose value is only known to lie within a certain interval $[\underline{\xi}, \bar{\xi}]$. Thus the remainder can be estimated using interval analysis. These methods are well developed (see Rall (1981)). For informations concerning the enclosure operations we direct to (Kaucher and Baumhof (1993), Stauning (1995)).

\section{Automatic differentiation}

Automatic differentiation avoids the drawbacks of numerical and symbolic differentiation. Roughly speaking in automatic differentiation the differentiation rules and the evaluation of formulas are carried out in parallel. So only real numbers have to be dealt with. The final result of automatic differentiation is the value of the derivative (even of a partial derivative) at some fixed point.

\section{Function evaluation}

Of great importance is to produce upper and lower bounds to exact values and ranges of values of real functions. The simplest way is to use a straight forward interval arithmetic evaluation. Reducing the number of occurences of a variable 
before interval evaluation leads to sharper bounds. Even better results can be obtained using centered or mean valued representation of functions (cf. Moore (1966), Lohner (1988)).

Finally we provide a concrete scheme, displaying all steps of the estimation algorithm, as shown in Table 1.

\section{Numerical Tests}

In this section we report on numerical experiments. The proposed algorithm works independent of the scheme employed in STEP 1, therefore we restrict for ease of simplicity to the trapezoidal method and Runge-Kutta method as underlying numerical methods for solving Volterra integral equations and ordinary initial value problems respectively. With these schemes we computed an approximation and display the (guaranteed) error bounds from the exact solution, which has been determined with the estimation algorithm. For simplicity the uniform stepsize of $3.90625 e-3$ has been used in examples 8.1-8.6. We display the solution at several selected points.

EXAMPLE 8.1. The first problem results from cancer chemotherapy (Feldmann and Schneider (1976)).

$$
\begin{aligned}
& x(s)=1-\int_{0}^{s} e^{-t^{2}} d t+\int_{0}^{s} e^{-(s-t)^{2}} x(t) d t, \quad 0 \leq s \leq 1 . \\
& \begin{array}{c|c}
s & \text { accuracy achieved by STEP } 9 \\
\hline 0.25 & 3.3 e-6 \\
0.50 & 5.7 e-6 \\
0.75 & 6.5 e-6
\end{array}
\end{aligned}
$$

EXAMPLE 8.2. This equation is taken from Linz (1985), pp 119.

$$
\begin{aligned}
& x(s)=e^{s}-\int_{0}^{s} e^{(s-t)} x(t) d t, \quad 0 \leq s \leq 1 . \\
& \begin{array}{c|c}
s & \text { accuracy achieved by STEP } 9 \\
\hline 0.25 & 2.6 e-6 \\
1.0 & 6.6 e-5
\end{array}
\end{aligned}
$$

Example 8.3. The following nonlinear Volterra integral equation is due to Tricomi (1955).

$$
x(s)=\cos (s)+\sin (s)+\int_{0}^{s} \sin (s-t)[x(t)]^{3} d t, \quad 0 \leq s \leq 1 .
$$


Table 1: Steps of the estimation algorithm

\begin{tabular}{|c|c|c|}
\hline STEP & OPERATION & ARITHMETIC \\
\hline 1 & $\begin{array}{l}\text { Given an initial value problem or integral equation. } \\
\text { Compute an approximation } \tilde{y} \text { at discrete points using } \\
\text { a suitable numerical scheme; }\end{array}$ & $\mathbb{I R}$ \\
\hline \multirow[t]{2}{*}{2} & $\begin{array}{l}\text { Formulate the problem as equivalent integral equation } \\
\text { with a Volterra integral operator } k\end{array}$ & IR \\
\hline & $y=g+k(y)=: f(y)$ & \\
\hline \multirow[t]{2}{*}{3} & Rewrite as root finding problem & $\mathbb{R}$ \\
\hline & $y-f(y)=0$ & \\
\hline \multirow[t]{2}{*}{4} & Apply the Newton operator $R^{-1}$ & IR \\
\hline & $y=R^{-1}\left(f(y)-\rho_{\eta}(y)\right)=T(y)$ & \\
\hline 5 & Compute enclosure $\tilde{Y}, G, K$ for $\tilde{y}, g, k$ & $I(I R)$ \\
\hline 6 & $\begin{array}{l}\text { Employ automatic differentiation to calculate an enclo- } \\
\text { sure } K_{u} \text { of } \frac{\partial k(s, t, u)}{\partial u}(\tilde{y})\end{array}$ & $I(I R)$ \\
\hline 7 & Determine range $[\underline{m}, \bar{m}]$ of $K_{u}$ & $I(I R)$ \\
\hline \multirow[t]{3}{*}{8} & $\begin{array}{l}\text { Establish }\left\|R^{-1}\right\| \leq \rho \text { with } \rho:=\exp |[\underline{m}, \bar{m}] a| \\
\text { Compute }[\underline{d}, \bar{d}] \text { the range of }\end{array}$ & $I(I R)$ \\
\hline & $\tilde{Y}(s)-G(s)-\int_{0} K(s, t) \tilde{Y}(t) d t$ & \\
\hline & Define $\delta:=|[\underline{d}, \bar{d}]|$ & \\
\hline 9 & Specify $V=[\underline{v}, \bar{v}] \in J_{n}$ by $0 \in V ;$ Define $\gamma:=\operatorname{diam}(V)$ & $I(I R)$ \\
\hline 10 & $\begin{array}{l}\text { Compute an enclosure } K_{u u}(v) \text { for } \\
\qquad \frac{\partial^{2} k(s, t, u)}{\partial u^{2}}(v)\end{array}$ & $I(I R)$ \\
\hline $\begin{array}{l}11 \\
12\end{array}$ & $\begin{array}{l}\text { Calculate } \psi^{*}:=\frac{1}{2}\left|K_{u u}(v)\right|\|V\|^{2} s \\
\text { Check } 0 \leq \delta \leq \frac{\gamma}{\rho}-\psi^{*} ; \\
\text { If YES: Solution validated; } \\
\text { If NO: Return to } 1 \text { and compute a better approximate } \\
\text { solution (e.g. by reducing the stepsize or taking another } \\
\text { numerical scheme) }\end{array}$ & $\begin{array}{l}I(\mathbb{I R}) \\
\mathbb{I R}\end{array}$ \\
\hline
\end{tabular}




\begin{tabular}{c|c}
$s$ & accuracy achieved by STEP 9 \\
\hline 0.25 & $1.0 e-4$ \\
0.75 & $9.4 e-2$
\end{tabular}

EXAmple 8.4. As linear Volterra integral equation of the first kind we consider (cf. Linz (1985))

$$
\int_{0}^{s} \cos (s-t) x(t) d t=1-\cos (s), \quad 0 \leq s \leq 1,
$$

with the techniques explained in section 5 , it is converted into a second kind equation.

\begin{tabular}{c|c}
$s$ & accuracy achieved by STEP 9 \\
\hline 0.25 & $4.8 e-6$ \\
0.50 & $9.7 e-6$ \\
0.75 & $1.4 e-5$ \\
1.0 & $1.8 e-5$
\end{tabular}

EXAMPLE 8.5. The differential equation of the harmonic oscillator is given by

$$
\begin{aligned}
& u^{\prime \prime}(x)+u(x)=0, \quad 0 \leq x \leq 1, \\
& u(0)=1, u^{\prime}(0)=0 \text {. } \\
& \begin{array}{c|c}
x & \text { accuracy achieved by STEP } 9 \\
\hline 0.25 & 6.6 e-6 \\
1.0 & 2.4 e-5
\end{array}
\end{aligned}
$$

EXAMPLE 8.6. We deal with the nonlinear initial value problem (see Grigorieff (1977))

$$
\begin{gathered}
u^{\prime}(x)=[u(x)]^{2}, \quad 0 \leq x \leq 1 \\
u(0)=-1
\end{gathered}
$$

\begin{tabular}{c|c}
$x$ & accuracy achieved by STEP 9 \\
\hline 0.5 & $1.1 e-5$ \\
1.0 & $2.6 e-6$
\end{tabular}

For the following partial differential equations the step sizes in $x$-direction resp. $y$-direction have been chosen as $1.25 e-1$ resp. $7.8125 e-3$, where central second order difference quotients have been used. 
EXAMPLE 8.7. The telegraph equation (cf. Collatz (1968)) has the form

$$
\begin{gathered}
-u_{x x}(x, y)+u_{y y}(x, y)=u(x, y), \quad 0 \leq x, y \leq 1, \\
u(x, 0)=0, \\
u_{y}(x, 0)=0 .
\end{gathered}
$$

\begin{tabular}{c|c|c}
$x$ & $y$ & accuracy achieved by STEP 9 \\
\hline 0.5 & 0.25 & $1.4 e-3$ \\
0.25 & 1.0 & $6.6 e-1$ \\
0.75 & 0.5 & $7.1 e-2$ \\
1.0 & 0.25 & $3.7 e-2$
\end{tabular}

EXAMPLE 8.8. As test problem for a nonlinear characteristic initial value problem we take (cf. Walter (1970)):

$$
\begin{gathered}
u_{x y}(x, y)=1+u(x, y)\left(u_{x}(x, y)+u_{y}(x, y)\right), \quad 0 \leq x, y \leq 1, \\
u(x, 0)=0, \\
u(0, y)=0 .
\end{gathered}
$$

\begin{tabular}{c|c|c}
$x$ & $y$ & accuracy achieved by STEP 9 \\
\hline 0.5 & 0.5 & $4.2 e-2$ \\
0.5 & 0.75 & $1.1 e-1$ \\
0.75 & 0.75 & $4.1 e-1$
\end{tabular}

\section{References}

Alefeld, G. and Herzberger, J. (1983): Introduction to Interval Computations. Academic Press, New York.

Burden, R. L. and Faires, J. D. (1993): Numerical Methods. PWS-Kent, Boston.

Collatz, L. (1968): Funktionalanalysis und numerische Mathematik. Springer, Berlin, Heidelberg, New York.

Dobner, H.-J. (1996): Bounds of high quality for first kind Volterra integral equations. Reliable Computing, 2(1), 33-45.

Dobner, H.-J. (1993): Verification Methods for Fredholm Integral Equations. International Journal of Computer Mathematics, Vol. 48, 251-261.

Feldmann, U. and Schneider, B. (1976): A General Approach to Multicompartment Analysis and Models for the Pharmacodynamics. Lecture Notes in Biomathematics, 11, 243-277.

Grigorieff, R. D. (1977): Numerik gewöhnlicher Differentialgleichungen 2. Teubner Stuttgart. 
Hammer, R., Hocks, M., Kulisch, U. and Ratz, D. (1993): Numerical Toolbox for Verified Computing I. Springer, Berlin, Heidelberg.

Kaucher, E., and Miranker, W. L. (1984): Self-Validating Numerics for Function Space Problems. Academic Press, New York.

Kaucher, E. and Baumhof, C. (1993): A Verified Computation of Fourier-Representations of Solutions for Functional Equations. Computing, Suppl. (9), 101-115.

Kulisch, U. and Miranker, W. L. (1981): Computer Arithmetic in Theory and Practice. Academic Press, New York.

Linz, P. (1985): Analytical and Numerical Methods for Volterra Equations. SIAM Philadelphia.

Lohner, R. (1988): Einschließung der Lösung gewöhnlicher Anfangs- und Randwertaufgaben. Dissertation, Karlsruhe.

Moore, R. E. (1966): Interval Analysis. Prentice Hall, Englewood Cliffs, New Jersey.

Nakao, M. T. (1988): A numerical approach to the proof of existence of solutions for elliptic problems. Japan Journal of Applied Mathematics, 5, 313-332.

Nakao, M. T. (1992): A numerical verification method for the existence of weak solutions for nonlinear boundary value problems. Journal of Mathematical Analysis and Applications, 164, 489-507.

Nakao, M. T. (1994): Numerical verifications of solutions for nonlinear hyperbolic equations. Interval Computations. 4, 64-77.

Neumaier, A. (1990): Interval Methods for Systems of Equations. Cambridge University Press, Cambridge.

Plum, M. (1994): Inclusion Methods for Elliptic Boundary Value Problems. In "Topics in Validated Computations", J. Herzberger (Editor). Elsevier Science B.V., Amsterdam, 323-379.

Rall, L. B. (1981): Automatic Differentiation. Springer, Berlin, Heidelberg.

Rump, S. M. (1993): Validated Solution of Large Linear Systems. Computing, Suppl. 9, 191-212.

Stauning, O. (1995): Solving Integral Equations Using Interval Analysis. Technical Report IMM-REP-1995-21, Technical University of Denmark, Lyngby.

Tricomi, F. G. (1955): Integral Equations. Dover, New York.

Walter, W. (1970): Differential and Integral Inequalities. Springer, Berlin, Heidelberg, New York.

Received September 25, 1997 